\title{
Article
}

\section{Scaling of phase diagram and critical point parameters in liquid-vapour phase transition of metallic fluids}

\author{
S. V. G. MENON ${ }^{1+*}$ \\ 1 Shiv Enclave, 304, 31-B-Wing, Tilak Nagar, Mumbai, India, 400089; menon.svg98@gmail.com \\ * Correspondence: menon.svg98@gmail.com; Tel.: +918879394488 \\ † Retired from Bhabha Atomic Research Centre, Mumbai, India, 400085
}

\begin{abstract}
The first objective of this paper is to investigate the scaling behavior of liquid-vapor phase transition in FCC and BCC metals starting from the zero-temperature four-parameter formula for cohesive energy. The effective potentials between the atoms in the solid are determined using lattice inversion techniques as a function of scaling variables in the above formula. These potentials are split into repulsive and attractive parts as per the Weeks-Chandler-Anderson prescription, and used in the coupling-parameter expansion for solving the Ornstein-Zernike equation supplemented with an accurate closure. Thermodynamic quantities obtained via the correlation functions are used to obtain critical point parameters and liquid-vapor phase diagrams. Their dependence on the scaling variables in the cohesive energy formula are also determined. Equally important second objective of the paper is to revisit coupling parameter expansion for solving the Ornstein-Zernike equation. The Newton-Armijo non-linear solver and Krylov-space based linear solvers are employed in this regard. These methods generate a robust algorithm that can be used to span the entire fluid region, except very low temperatures. Accuracy of the method is established by comparing the phase diagrams with those obtained via computer simulation. Avoidance of the 'no-solution-region' of Ornstein-Zernike equation in coupling-parameter expansion is also discussed. Details of the method and the complete algorithm provided here would make this technique more accessible to researchers investigating thermodynamic properties of one component fluids.
\end{abstract}

Keywords: Liquid-vapour phase transition; metals; thermodynamic perturbation theory; coupling-parameter expansion; critical point parameters; universal aspects; scaled variables.

\section{Introduction}

Scaling and universal features in phase transition theory were first brought out with the van der Waals equation of state [1]. Scaling the thermodynamic variables provided a universal equation of state and critical behavior characterized via universal critical exponents. The mean field approach implied in the van der Waals equation neglects long range fluctuations close to the critical point and so provide only the classical critical behavior as opposed to the renormalizatioin group theory of critical phenomena [2]. The Ornstein-Zernike equation (OZE) defines the relationship between short range and long range correlation functions in fluids. When supplemented with appropriate closure relations, which relates the correlation functions to inter-particle interaction, OZE provides a framework to employ the mean field theory to fluids with arbitrary inter molecular potentials [3]. Thermodynamic properties with accuracy comparable to those obtained via simulation are obtained presently with the OZE.

The zero-temperature cohesive energy for a variety of metals follow a universal curve with suitable scaled variables [4]. It is then natural to explore if the universal energy- volume curve can be extended to the expanded volume states to describe the liquid-vapor phase transition in metallic fluids [5]. This investigation becomes easy when used with the corrected rigid spheres (CRIS) model, which is a thermodynamic perturbation theory based on the cohesive energy of nearest-neighbor pairs in the 
fluid [6]. In comparison to other perturbation theories [7], which are based on inter-particle interaction potentials, CRIS model provides an approximate theory of fluids based on cohesive energy curves. However, accurate prediction of liquid-vapor phase diagram within the CRIS model needs tuning of reference-hard-sphere pair distribution function [8].

The first objective in this paper is to investigate the scaling aspects of liquid-vapor phase transition in metallic fluids starting from the scaled cohesive energy (SCE) versus volume curves. An improved SCE formula [9], involving four-parameters, is used for this purpose. First of all, effective pair interaction potentials are derived from the SCE formula by employing lattice inversion techniques, and split into repulsive and attractive components using the Weeks-Chandler-Anderson (WCA) prescription [11]. These components are used in an accurate thermodynamic perturbation theory, called coupling-parameter expansion (CPE) [10], for solving the Ornstein-Zernike equation (OZE) and an appropriate closure relation. The coupling parameter $(0 \leq \lambda \leq 1)$ tunes the strength of the attractive component of the potential, and all correlation functions are expressed as Taylor's series in $\lambda$ around $\lambda=0$. It is important that the method offers series expansion to arbitrary orders in Taylor's series. Therefore, all the thermodynamic quantities are easily computed in the entire phase plane once the correlation functions are determined to sufficient accuracy. The critical point parameters and phase diagrams of metallic fluids are then obtained in terms of the scaling variables in the SCE formula. Results for critical point parameters are similar to those obtained earlier [5] using the CRIS model, however there are important differences. For example, the FCC and BCC lattices are found to generate different type of critical point parameters. Further, scaling of phase diagrams was not considered earlier.

The second objective of the paper is to revisit, in some detail, the methods to be used for implementing the CPE technique. It needs the correlation functions given by the non-linear OZE for a reference system, and solutions to a hierarchy of linear integral equations for derivatives of the correlation functions. It is most appropriate to use the Newton-Armijo non-linear solver and Krylov space-based linear solvers [12] for this purpose. In addition to facilitating computation of correlation functions to high order perturbation theory, the CPE technique avoids the 'no-solution region' of the OZE in the liquid-vapor transition region. Occurrence of such regions, for the hypernetted chain (HNC) closure [13] and other closure relations [14], is indeed a bottleneck in applying OZE with the full potential.

The following sections discuss different aspects of the paper such as the SCE versus volume formula, corrections to be applied for small volumes, lattice inversion to derive potentials, and algorithmic details of the CPE technique. Simulation results on phase diagrams, taken from literature, for specific potentials are compared with results obtained using present method to establish its accuracy. Critical point parameters and phase diagrams are obtained for potentials corresponding to FCC and BCC lattices. Details of the complete algorithm for easy implementation of CPE are provided in the appendix.

\section{Scaled cohesive energy formula}

The Fermi-pressure in degenerate electron systems, which is a pure quantum effect, manifests as the zero-temperature isotherm in metals. This contributes significantly to total pressure in compressed solids, and becomes the dominant component at strong compression. Density functional theories (DFT) are routinely used now [15] to generate energy versus specific volume (or volume per atom) tables, which are then easily incorporated into semi-empirical formula. Such a formulation for zero-temperature energy and pressure, involving four-parameters [9], is expressed in the SCE formula:

$$
\begin{aligned}
E_{c}(\mathcal{V}) & =-\mathcal{E}_{0}\left(1+a+\delta a^{3}\right) e^{-a} \\
P_{c}(\mathcal{V}) & =3 B_{0}\left(1-\left(\mathcal{V} / \mathcal{V}_{0}\right)^{1 / 3}\right)\left(\mathcal{V} / \mathcal{V}_{0}\right)^{-2 / 3}\left(1-3 \delta a+\delta a^{2}\right) e^{-a} \\
\eta & =\left(9 B_{0} \mathcal{V}_{0} / E_{c o h}\right)^{1 / 2}, a=\eta\left(\left(\mathcal{V} / \mathcal{V}_{0}\right)^{1 / 3}-1\right), \delta=(1 / 2)\left(B_{0}^{\prime}-1\right) / \eta-1 / 3
\end{aligned}
$$


The four parameters in this model are the atomic volume $\mathcal{V}_{0}$, the bulk modulus $B_{0}$, its pressure derivative $B_{0}^{\prime}$ and the cohesive energy per atom $\mathcal{E}_{0}$ at equilibrium conditions. This is a refinement over Rose equation [4], and provides accurate zero-temperature energy and pressure in compressed states up to $\sim \mathcal{V}_{0} / 2$, (which corresponds to about $150 \mathrm{GPa}$ ), and expanded states up to about $\sim 2 \mathcal{V}_{0}$ for about forty metals [9]. If energy $E_{c}$ and pressure $P_{c}$ are scaled with $\mathcal{E}_{0}$ and $B_{0}$, respectively, there remains only two dimensionless parameters $\eta$ and $\delta$ in these expressions. Note that the parameter ' $a$ ' is related to the dimensionless length variable $\left(\mathcal{V} / \mathcal{V}_{0}\right)^{1 / 3}$, which is simply the (scaled) side length of the atomic volume. Unless specified explicitly, length and energy will be scaled with $\mathcal{V}_{0}^{1 / 3}$ and $\mathcal{E}_{0}$, respectively, throughout. Negative ' $a$ ' corresponds to compressed states while it is positive for the expanded states. Furthermore, the equilibrium atomic volume $\mathcal{V}_{0}$ is slightly lower than its value at ambient conditions, viz., $T_{0}=300 \mathrm{~K}$ and $P_{0}=1$ bar. It should be determined such that the zero-temperature pressure, together with thermal pressure of ions and electrons is just one bar at $300 \mathrm{~K}$.

\subsection{Correction for strong compression}

The expressions given above are inadequate in the region of strong compression. This is evident from Eq.(1), which approaches a finite value as $\mathcal{V} \rightarrow 0$. In this limit, energy and pressure must approach those of an electron gas around the nucleus, as given by the quantum statistical model (QSM) [16]. This model provides accurate electronic properties above $\sim 250 \mathrm{GPa}$ of pressure, as it accounts for exchange and correlation effects in addition to incorporating corrections for electron density gradients [17]. Electron pressure in a compressed atom within the QSM model is analytically expressed as:

$$
\begin{aligned}
& P_{q}(\mathcal{V})=\frac{\hbar^{2}}{2 m_{e}}\left[\frac{2}{5}\left(3 \pi^{2}\right)^{2 / 3} N_{s}^{5 / 3}-\frac{2}{a_{B}} \frac{13}{36}(3 / \pi)^{1 / 3} N_{s}^{4 / 3}\right], \\
& N_{s}(\mathcal{V})=\frac{Z_{n}}{\mathcal{V}} \operatorname{Exp}\left[-\alpha\left(\frac{\mathcal{V}}{\mathcal{V}_{0}}\right)^{1 / 3}-\beta\left(\frac{\mathcal{V}}{\mathcal{V}_{0}}\right)^{2 / 3}\right] \text {, } \\
& \alpha=0.1935 Z_{n}^{\left[0.495-0.039\left(\log _{10} Z_{n}\right)\right]} \frac{R_{w}}{a_{B}}, \\
& \beta=\left[0.068+0.078\left(\log _{10} Z_{n}\right)-0.086\left(\log _{10} Z_{n}\right)^{2}\right]\left(\frac{R_{w}}{a_{B}}\right)^{2} .
\end{aligned}
$$

Here, $\hbar$ is reduced Planck's constant, $m_{e}$ electron mass, $a_{B}$ Bohr radius, $Z_{n}$ atomic number and $R_{w}$ the equilibrium Wigner-Seitz radius. The quantity $N_{S}(\mathcal{V})$ is electron density at the atomic cell surface, and the negative contribution in pressure is due to exchange effect. This contribution extends the range of validity of $P_{q}(\mathcal{V})$ to larger $\mathcal{V}$. The internal energy per atom, $E_{q}(\mathcal{V})$, is obtained by integrating the thermodynamic relation $P=-d E / d V$ from a suitable initial volume, say $\mathcal{V}_{0}$.

It is easy to use an interpolation procedure [18] to smoothly go from SCE model to QSM. Choose a volume $\mathcal{V}_{m}$ such that the SEC model is accurate for $\mathcal{V} \geq \mathcal{V}_{m}$, so that the corrected zero temperature pressure and energy are, respectively, $P_{0}(\mathcal{V})=P_{c}(\mathcal{V})$ and $E_{0}(\mathcal{V})=E_{\mathcal{c}}(\mathcal{V})$ for $\mathcal{V} \geq \mathcal{V}_{m}$. The interpolated expressions for smaller volumes are expressed as:

$$
\begin{aligned}
& E_{0}(\mathcal{V})=\left[E_{q}(\mathcal{V})-E_{q}\left(\mathcal{V}_{m}\right)\right] B_{i}(\mathcal{V})+E_{c}\left(\mathcal{V}_{m}\right), \quad \mathcal{V} \leq \mathcal{V}_{m} \\
& P_{0}(\mathcal{V})=P_{q}(\mathcal{V}) B_{i}(\mathcal{V})+\left[E_{q}(\mathcal{V})-E_{q}\left(\mathcal{V}_{m}\right)\right] B_{i}^{\prime}(\mathcal{V}), \quad \mathcal{V} \leq V_{m}
\end{aligned}
$$

where $B_{i}(\mathcal{V})=\left[1+b_{1} \mathcal{V}+b_{2} \mathcal{V}^{4 / 3}+b_{3} \mathcal{V}^{5 / 3}\right]$ is an interpolating function. The parameters $b_{1}, b_{2}$ and $b_{3}$ are chosen such that $P_{0}(\mathcal{V})$ and its first two derivatives are continuous at $\mathcal{V}_{m}$ [18]. Note that $E_{0}(\mathcal{V})$ is continuous at $\mathcal{V}_{m}$ by definition. This procedure gives a smooth transition from SCE model to the QSM, and is expected to be better than modifying the definition of $E_{\mathcal{c}}(\mathcal{V})$ arbitrarily [19].

\section{Lattice inversion for potential}

The lattice inversion method [20] is a convenient tool to extract effective inter-particle potentials from $E_{0}(\mathcal{V})$, which is re-expressed in dimensionless functional form as $E(x)$. More specifically, $E(x)=$ 
$E_{0}\left(\mathcal{V}_{0} x^{3}\right) / \mathcal{E}_{0}$, where $x=\left(\mathcal{V} / \mathcal{V}_{0}\right)^{1 / 3}$ is the scaled side length of the atomic volume. The lattice is imagined as an assembly of successive shells around a central atom, and so $E(x)$ is expressed as a lattice sum over inter-particle potential $U(r)$ where $r$ is the (scaled) nearest-neighbor distance (NND) :

$$
E(x)=\frac{1}{2} \sum_{n=1}^{\infty} \gamma_{n} U\left(b_{n} z_{0} x\right) .
$$

Here $n$ is the shell index, $\gamma_{n}$ number of atoms on the shell, $b_{n}$ the normalized shell-radius $\left(b_{1}=1\right)$ in units of NND. The factor $1 / 2$ in this equation arises as $U(r)$ is defined for a pair of atoms while $E(x)$ corresponds to a single atom. For numerical applications, the infinite sum is truncated at some finite shell $(\sim 20)$ so that $U$ is negligible there after. The NND and $x$ are related as $r=z_{0} x$, where $z_{0}$ is a constant dependent on the lattice type. For the FCC lattice (four atoms in unit cell) $z_{0}=2^{1 / 6}$, while for BCC lattice (two atoms in unit cell) $z_{0}=\sqrt{3} / \sqrt[3]{4}$. On separating the first shell-contribution, Eq.(6) is expressed as:

$$
U(r)=\frac{2}{\gamma_{1}} E\left(r / z_{0}\right)-\frac{1}{\gamma_{1}} \sum_{m=2}^{\infty} \gamma_{m} U\left(b_{m} r\right)
$$

The subtracted term is the contribution to $U(r)$ from second and higher shells. This equation is solved via iteration [21] to express $U(r)$ explicitly in terms of $E(x)$ [20]. However, for computational purposes, it is reasonable to assume that $U(r)=0$ for $r \geq r_{\infty}$ for a sufficiently large value of $r_{\infty}$. Then, Eq.(7) is solved [21] by marching to lower values of $r$ starting from $r_{\infty}$, as the second term is already computed. It is advantageous to define a geometrically progressing mesh over the required domain, $r_{1} \leq r \leq r_{\infty}$, and interpolate $U(r)$ for in-between values of $r$ for computing the second term. Alternatively, starting with the approximation provided by the first term, few iterations on the second term readily provides a converged profile of $U(r)$.

Table 1. Shell radii and occupation numbers for FCC lattice.

\begin{tabular}{cccccccccccc}
\hline $\mathbf{n}$ & $\gamma_{n}$ & $b_{n}$ & $\mathbf{n}$ & $\gamma_{n}$ & $b_{n}$ & $\mathbf{n}$ & $\gamma_{n}$ & $b_{n}$ & $\mathbf{n}$ & $\gamma_{n}$ & $b_{n}{ }^{1}$ \\
\hline 1 & 12 & 1 & 6 & 8 & $\sqrt{6}$ & 11 & 24 & $\sqrt{11}$ & 16 & 48 & $\sqrt{17}$ \\
2 & 6 & $\sqrt{2}$ & 7 & 48 & $\sqrt{7}$ & 12 & 24 & $\sqrt{12}$ & 17 & 30 & $\sqrt{18}$ \\
3 & 24 & $\sqrt{3}$ & 8 & 6 & $\sqrt{8}$ & 13 & 72 & $\sqrt{13}$ & 18 & 72 & $\sqrt{19}$ \\
4 & 12 & 2 & 9 & 36 & 3 & 14 & 48 & $\sqrt{15}$ & 19 & 24 & $\sqrt{20}$ \\
5 & 24 & $\sqrt{5}$ & 10 & 24 & $\sqrt{10}$ & 15 & 12 & 4 & 20 & 48 & $\sqrt{21}$ \\
\hline
\end{tabular}

${ }^{1}$ Note that $b_{14}=\sqrt{15}$. There is no shell of radius $\sqrt{14}$.

Tables for the lattice dependent constants have been enumerated [22] for 50 shells. In fact, tabulations are provided for elementary sub-lattices where atoms are placed only at cell corners (SC), faces centers (FC), and edge centers (EC). All the cubic lattices (FCC, BCC, ECC, etc.) are decomposed into these sub-lattice types. The lattice dependent constants $\gamma_{n}$ and $b_{n}$ for $1 \leq n \leq 20$ for FCC and BCC lattices are reproduced in Table 1 and Table 2, respectively, for convenience.

Table 2. Shell radii and occupation numbers for BCC lattice.

\begin{tabular}{cccccccccccc}
\hline $\mathbf{n}$ & $\gamma_{n}$ & $b_{n}$ & $\mathbf{n}$ & $\gamma_{n}$ & $b_{n}$ & $\mathbf{n}$ & $\gamma_{n}$ & $b_{n}$ & $\mathbf{n}$ & $\gamma_{n}$ & $b_{n}{ }^{1}$ \\
\hline 1 & 8 & 1 & 6 & 6 & $4 / \sqrt{3}$ & 11 & 12 & $4 \sqrt{2 / 3}$ & 16 & 24 & $2 \sqrt{11 / 3}$ \\
2 & 6 & $2 / \sqrt{3}$ & 7 & 24 & $\sqrt{19 / 3}$ & 12 & 48 & $\sqrt{35 / 3}$ & 17 & 8 & 4 \\
3 & 12 & $2 \sqrt{2 / 3}$ & 8 & 24 & $2 \sqrt{5 / 3}$ & 13 & 30 & $2 \sqrt{3}$ & 18 & 48 & $\sqrt{17}$ \\
4 & 24 & $\sqrt{11 / 3}$ & 9 & 24 & $2 \sqrt{2}$ & 14 & 24 & $2 \sqrt{10 / 3}$ & 19 & 24 & $2 \sqrt{13 / 3}$ \\
5 & 8 & 2 & 10 & 32 & 3 & 15 & 24 & $\sqrt{43 / 3}$ & 20 & 48 & $2 \sqrt{14 / 3}$ \\
\hline
\end{tabular}

${ }^{1}$ Note that there is no formula for radii in BCC lattice. 


\subsection{Chen-Möbius formula}

A remarkable explicit formula for the potential in terms of $E(x)$ is obtained [23] by generalizing the lattice sum expression to the form:

$$
E(x)=\frac{1}{2} \sum_{n=1}^{\infty} \Gamma_{n} U\left(B_{n} z_{0} x\right)
$$

Here, the expanded sequence of shell radii $\left\{B_{n}\right\}$ monotonically increses $\left(B_{1}=b_{1}=1\right)$, and contains the original sequence $\left\{b_{n}\right\}$. Most importantly, the expanded sequence has the additional property that it forms a semi-group. That is, for any two integers $n$ and $m$, there exist an integer $p$ such that $B_{p}=B_{n} B_{m}$. The elements of $\left\{B_{n}\right\}$ which are not contained in the original set $\left\{b_{n}\right\}$ are radii of virtual shells, and the corresponding occupation numbers in the expanded set $\left\{\Gamma_{n}\right\}$ are zero. Thus for the FCC lattice, the expanded radii are given by: $B_{n}=\sqrt{n}$. So 14 is a virtual shell and $\Gamma_{14}=0$ as $B_{14}=\sqrt{14}$ is not in the original set $\left\{b_{n}\right\}$ (see Table 1 ). For the BCC lattice, the set $\left\{B_{n}\right\}$ is to be generated appropriately from $\left\{b_{n}\right\}$ so as to have the semi-group property. Now, motivated by Eq.(7), a general expansion for $U(r)$ is attempted in the form:

$$
U(r)=2 \sum_{m=1}^{\infty} I_{m} E\left(B_{m} r / z_{0}\right)
$$

where $\left\{I_{m}\right\}$ are weight factors for different shells. Substituting this expansion in Eq.(8) gives:

$$
E(x)=\sum_{n=1}^{\infty} \Gamma_{n} \sum_{m=1}^{\infty} I_{m} E\left(B_{m} B_{n} x\right)
$$

The double sum on the right-hand-side can be rewritten by grouping terms using the semi-group prperty. Substituting $B_{m} B_{n}=B_{p}$, and grouping terms yields:

$$
E(x)=\sum_{p=1}^{\infty} E\left(B_{p} x\right) \sum_{m=1}^{p} I_{m} \Gamma_{m[p]} \delta\left[B_{p}-B_{m} B_{m[p]}\right] .
$$

Here, $\delta[x-y]$ equals 1 for $x=y$ and 0 for $x \neq y$. For a given $p$, the indices $m$ and $m[p]$ are those satisfying the semi-group property. The second sum terminates at $p$ due to the monotonic property of the elements in $\left\{B_{p}\right\}$. Now, Eq.(11) reduces to an identity if $\left\{I_{p}\right\}$ are chosen so that the second sum is 1 for $p=1$ and 0 for $p \geq 2$. A recursion formula for $I_{p}$, for $p \geq 2$, then follows on separating out the last term in this sum:

$$
I_{1}=\frac{1}{\Gamma_{1}} ; \quad I_{p}=-\frac{1}{\Gamma_{1}} \sum_{m=1}^{p-1} I_{m} \Gamma_{m[p]} \delta\left[B_{p}-B_{m} B_{m[p]}\right], \quad p \geq 2 .
$$

Note that $m[p]=p$ for $p=1$. Thus the weight factors $\left\{I_{p}\right\}$ in Eq.(9) are readily computed once $\left\{\Gamma_{n}\right\}$ and $\left\{B_{n}\right\}$ are specified. This choice is already discussed for FCC lattice, and the generated inversion-constants are given in Table 3 for 20 shells. Note that shell 14 is virtual and $\Gamma_{14}=0$. 
Table 3. Constants of Chen-Möbius inversion formula for FCC lattice.

\begin{tabular}{cccccccccccccccc}
\hline $\mathbf{n}$ & $\Gamma_{n}$ & $B_{n}$ & $I_{n}$ & $\mathbf{n}$ & $\Gamma_{n}$ & $B_{n}$ & $I_{n}$ & $\mathbf{n}$ & $\Gamma_{n}$ & $B_{n}$ & $I_{n}$ & $\mathbf{n}$ & $\Gamma_{n}$ & $B_{n}$ & $I_{n}{ }^{1}$ \\
\hline 1 & 12 & $\sqrt{1}$ & $1 / 12$ & 6 & 8 & $\sqrt{6}$ & $1 / 9$ & 11 & 24 & $\sqrt{11}$ & $-1 / 6$ & 16 & 24 & $\sqrt{16}$ & $-1 / 64$ \\
2 & 6 & $\sqrt{2}$ & $-1 / 24$ & 7 & 48 & $\sqrt{7}$ & $-1 / 3$ & 12 & 24 & $\sqrt{12}$ & $7 / 72$ & 17 & 24 & $\sqrt{17}$ & $-1 / 3$ \\
3 & 24 & $\sqrt{3}$ & $-1 / 6$ & 8 & 6 & $\sqrt{8}$ & $1 / 32$ & 13 & 72 & $\sqrt{13}$ & $-1 / 2$ & 18 & 24 & $\sqrt{18}$ & $-17 / 72$ \\
4 & 12 & $\sqrt{4}$ & $-1 / 16$ & 9 & 36 & $\sqrt{9}$ & $1 / 12$ & 14 & 0 & $\sqrt{14}$ & $1 / 3$ & 19 & 24 & $\sqrt{19}$ & $-1 / 2$ \\
5 & 24 & $\sqrt{5}$ & $-1 / 6$ & 10 & 24 & $\sqrt{10}$ & 0 & 15 & 48 & $\sqrt{15}$ & $1 / 3$ & 20 & 24 & $\sqrt{20}$ & $5 / 24$ \\
\hline
\end{tabular}

${ }^{1}$ Note that $B_{14}=\sqrt{14}$ and $\Gamma_{14}=0$ are introduced.

Generating a new set of shell radii $\left\{B_{n}\right\}$ and consequently $\left\{\Gamma_{n}\right\}$ is more involved in the case of BCC lattice. A possible approach [23] is to add virtual shells of radii $\left\{b_{2} b_{2}^{j}, b_{3} b_{2}^{j}, b_{4} b_{2}^{j}, \cdots\right.$, for $j=$ $1,2, \cdots\}$ to the existing set $\left\{b_{n}\right\}$. The occupation numbers $\Gamma_{n}$ of these new shells are zero. Then $\left\{B_{n}\right\}$ is generated by reordering the enlarged sets to obtain monotonically increasing shell radii which also satisfy the semi-group property. This procedure is not unique and several virtual shells are introduced as seen in Table 4 and Table 5. These tables, which cover a total of 30 shells, have 19 virtual shells. Nevertheless, the method provides an inversion formula as its parameters for sufficient number of shells are easily generated on a computer.

Table 4. Constants of Chen-Möbius inversion formula for BCC lattice.

\begin{tabular}{llllllllllll}
\hline $\mathbf{n}$ & $\Gamma_{n}$ & $B_{n}$ & $I_{n}$ & $\mathbf{n}$ & $\Gamma_{n}$ & $B_{n}$ & $I_{n}$ & $\mathbf{n}$ & $\Gamma_{n}$ & $B_{n}$ & $I_{n}{ }^{1}$ \\
\hline 1 & 8 & 1 & 0.12500 & 6 & 0 & 1.77778 & 0.03955 & 11 & 0 & 2.17732 & -0.31641 \\
2 & 6 & 1.15470 & -0.0975 & 7 & 0 & 1.88562 & 0.28125 & 12 & 0 & 2.21108 & 0.5625 \\
3 & 0 & 1.33333 & 0.07031 & 8 & 24 & 1.91485 & -0.375 & 13 & 6 & 2.30940 & 0.09375 \\
4 & 0 & 1.53960 & -0.05273 & 9 & 8 & 2.0 & -0.125 & 14 & 0 & 2.37037 & 0.02225 \\
5 & 12 & 1.63299 & -0.1875 & 10 & 0 & 2.05280 & -0.02966 & 15 & 0 & 2.51416 & 0.31641 \\
\hline
\end{tabular}

${ }^{1}$ Note that $B_{3}=1.33333$ and $\Gamma_{3}=0$, and many others are introduced.

Table 5. Constants of Chen-Möbius inversion formula for BCC lattice - continued.

\begin{tabular}{llllllllllll}
\hline $\mathbf{n}$ & $\Gamma_{n}$ & $B_{n}$ & $I_{n}$ & $\mathbf{n}$ & $\Gamma_{n}$ & $B_{n}$ & $I_{n}$ & $\mathbf{n}$ & $\Gamma_{n}$ & $B_{n}$ & $I_{n}{ }^{1}$ \\
\hline 16 & 24 & 2.51661 & -0.375 & 21 & 24 & 2.82843 & -0.375 & 26 & 32 & 3.0 & -0.5 \\
17 & 0 & 2.55314 & -0.63281 & 22 & 0 & 2.9031 & -0.29663 & 27 & 0 & 3.0792 & -0.58008 \\
18 & 24 & 2.58199 & -0.375 & 23 & 0 & 2.90593 & 0.5625 & 28 & 0 & 3.16049 & 0.01251 \\
19 & 0 & 2.66667 & 0.21094 & 24 & 0 & 2.94811 & 0.63281 & 29 & 12 & 3.26599 & 0.75 \\
20 & 0 & 2.73707 & -0.01669 & 25 & 0 & 2.98142 & 0.5625 & 30 & 0 & 3.35221 & 0.26697 \\
\hline
\end{tabular}

${ }^{1}$ Note that $B_{17}=2.55314$ and $\Gamma_{17}=0$, and many others are new.

\section{Inter-particle potentials for FCC and BCC metals}

The cohesive energy formula for metals is more general than in Eq.(6) as it is necessary to account for embedding energy [24] due to the presence of free electrons at lattice sites. The generalized form is given by:

$$
E_{\text {emb }}(x)=F(\psi)+\frac{1}{2} \sum_{n=1}^{\infty} \gamma_{n} U\left(b_{n} z_{0} x\right) .
$$

Here $F(\psi)$ is the embedding energy resulting due to the electrons, and $\psi$ is the electron density at the central atom. This electron density is expressible as a sum of the density $\left(\psi_{a}\left(r_{j}\right)\right)$ contributions from all other atoms, that is, $\psi=\sum_{j \neq 0} \psi_{a}\left(r_{j}\right)$. By employing suitable empirical forms for $F(\psi)$, its contribution from $E_{e m b}$ is readily subtracted out before determining the inter-particle potentials. However, such 
potentials would be devoid of the binding effects modeled as embedding energy. On the other hand, approximating $F(\psi)$ with a Taylor's expansion (truncated to second order) about an average electron density $\bar{\psi}$, Eq.(13) is reducible to the pair-potential form [26], however, with an effective potential given by $U_{e f f}(r)=U(r)+2 F^{\prime}(\bar{\psi}) \psi_{a}(r)+F^{\prime \prime}(\bar{\psi}) \psi_{a}^{2}(r)$. Furthermore, it is also found that the radial distribution functions of liquid metals obtained with the effective potentials agree quite well with simulation results using full potentials [26]. So it will be assumed here after, although not indicated explicitly, that all potentials derived from cohesive energy are effective potentials.
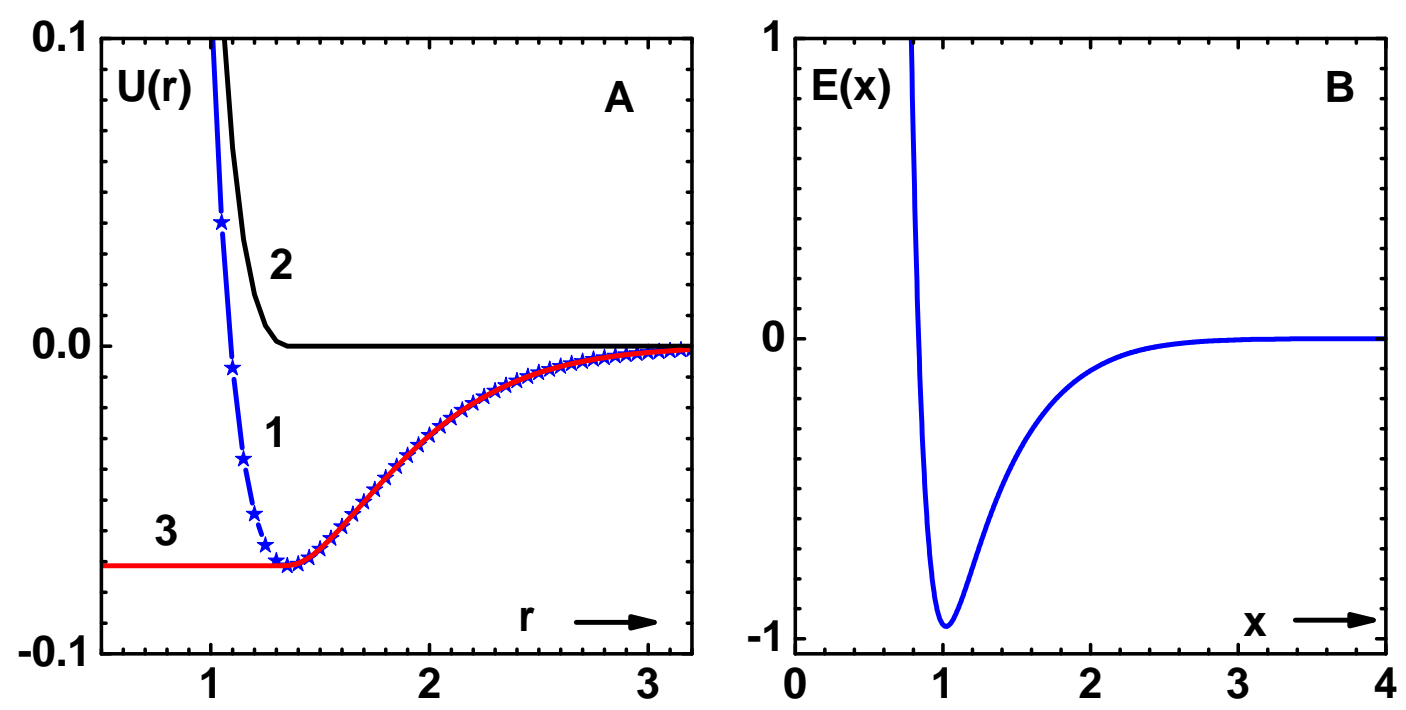

Figure 1. (A) Effective potential $U(r)$ for $C u$ versus scaled nearest-neighbor distance (curve-1), with repulsive (curve-2) and attractive components (curve-3) as per WCA prescription. Potential minimum is $U_{\text {min }}=-0.07143$ at $r_{\text {min }}=1.359$ in reduced units. (B) Cohesive energy $E(x)$ for Cu versus scaled side length of atomic volume $x$. Energy minimum (-1) occurs at (1) in reduced units. Length and energy units are $\mathcal{L}_{0}=\mathcal{V}_{0}{ }^{1 / 3}$ and $\mathcal{E}_{0}$ (see text), respectively.

Table 6. Material Parameters for four-parameter model .

\begin{tabular}{llllllllll}
\hline Material & $\mathcal{V}_{0}$ & $\begin{array}{l}B_{0} \\
\mathrm{GPa}\end{array}$ & $B_{0}^{\prime}$ & $\begin{array}{l}\mathcal{E}_{0} \\
\mathrm{eV}\end{array}$ & $\eta$ & $\delta$ & $\begin{array}{l}\mathcal{L}_{0} \\
\mathrm{~A}\end{array}$ & $\begin{array}{l}\text { At. No. } \\
Z_{n}\end{array}$ & $\begin{array}{l}\text { At. Wt. } \\
M_{n}\end{array}$ \\
\hline Copper & 11.38 & 134.8 & 5.19 & 3.489 & 5.0619 & 0.0808 & 2.2771 & 29 & 63.50 \\
Aluminum & 16.35 & 79.3 & 4.37 & 3.389 & 4.6361 & 0.0296 & 2.5382 & 13 & 26.98 \\
Gold & 16.95 & 180.7 & 5.43 & 3.812 & 6.7182 & -0.0034 & 2.5686 & 79 & 196.97 \\
Iron & 11.81 & 163.0 & 4.50 & 4.281 & 5.0270 & 0.0148 & 2.2775 & 26 & 55.85 \\
Tungsten & 15.82 & 325.0 & 4.36 & 8.791 & 5.732 & -0.0402 & 2.2775 & 74 & 183.85 \\
\hline
\end{tabular}

As an illustration of the model, the effective potential $U(r)$ versus nearest-neighbor distance $r$ for $\mathrm{Cu}$ (curve-1) is shown in Fig.1-A. The parameters used in the model are as given in Table 6. The potential minimum $U_{\min }=-0.07143$ occurs at $r_{\min }=1.359$ in reduced units. The repulsive (curve-2) and attractive (curves-3) components of the potential, as per WCA specification (see below), are also shown. The cohesive energy $E(x)$ versus side length $(x)$ of atomic volume is shown in Fig.1-B. Keeping all parameters except $\eta$ fixed, variations of the potential minimum $U_{\min }$ and position $r_{\min }$ versus $\eta$ are shown in Fig.2-A. These results, which correspond to FCC lattices in general, show that potential parameters are somewhat insensitive to $\eta$ beyond $\eta \sim 6$. Therefore, critical point parameters, to be discussed below, also would show this weak dependence for larger values of $\eta$. Similar results, 
obtained using data for Fe (see Table 6), which correspond to BCC lattices, are shown in Fig.2-B. The trends of variation of the parameters are similar, although the range of variation of $r_{\min }$ is much less.
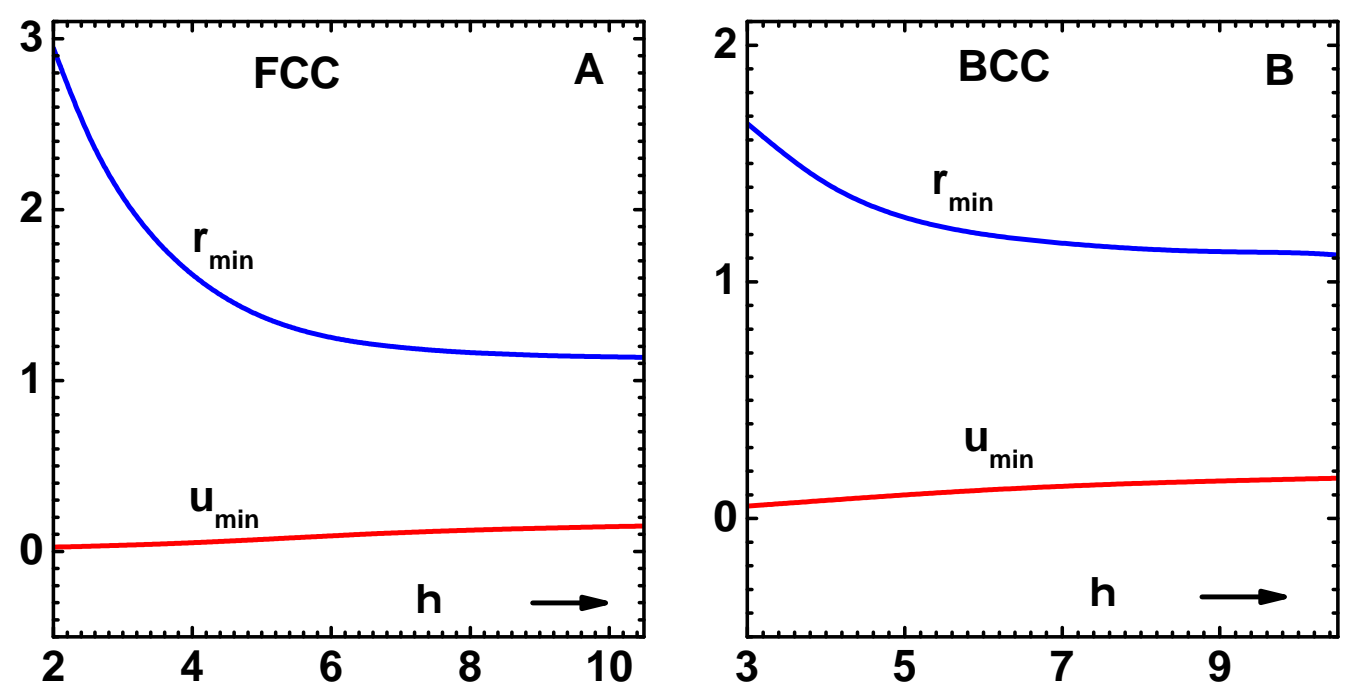

Figure 2. (A) Potential minimum $U_{\min }$ and its position $r_{\min }$ versus $\eta$ for FCC lattices. All other parameters correspond to those of $\mathrm{Cu}$ (see text). (B) Similar results for BCC lattices. All parameters except $\eta$ corresponds to those of Fe (see text).

Having discussed the details of obtaining the inter-particle potentials, it is now appropriate to consider a general method for computing properties of metallic fluids. Critical point parameters and phase diagrams in liquid-vapor phase transition are obtained thereafter. The scaling of cohesive energy and potential would imply such a scaling of critical point parameters with respect to $\eta$ and some (approximate) universal form for phase diagrams.

\section{Coupling-parameter expansion}

The CPE in thermodynamic perturbation theory[10] is based on dividing the inter-particle potential $U(r)$ into a reference (repulsive) and perturbation (attractive) components. The most appropriate division is based on the WCA prescription [11], which is defined as

$$
\begin{aligned}
& U_{R}(r)=U(r)-U_{\text {min }}, \quad r \leq r_{\text {min }}, \quad U_{R}(r)=0, \quad r>r_{\text {min }} \\
& U_{A}(r)=U_{\text {min }}, \quad r \leq r_{\text {min }}, \quad U_{A}(r)=U(r), \quad r>r_{\text {min }} .
\end{aligned}
$$

The reference part is purely repulsive and determines the structure of dense liquids while the attractive perturbing component is responsible for cohesion and phase changes [11]. A coupling-parameter $\lambda$ is introduced into the potential and it is expressed as $U(r, \lambda)=U_{R}(r)+\lambda U_{A}(r)$. Its magnitude $0 \leq \lambda \leq 1$ determines the strength of the perturbation; $U(r, 0)$ being the reference part and $U(r, 1)$ the full potential. All correlation functions, which determine the structure of the fluid, like the pair distribution function $g(r, \lambda)$, are now to be treated as functions of $\lambda$. In the thermodynamic perturbation theory (TPT), these functions are expanded as Taylor's series in $\lambda$ about their reference part as

$$
g(r, \lambda)=g_{0}(r)+\lambda g_{1}(r)+\cdots+\frac{1}{n !} \lambda^{n} g_{n}(r)+\cdots .
$$

Here $g_{0}(r)$ is the reference system's pair distribution function and $g_{n}(r)$ denotes its $n^{\text {th }}$ derivatives at $\lambda=0$. Similar Taylor's series representations hold for other correlation functions as well: the 
direct correlation function $c(r, \lambda)$, the total correlation function $h(r, \lambda)=g(r, \lambda)-1$, and the indirect correlation function $y(r, \lambda)=h(r, \lambda)-c(r, \lambda)$. For all of them, the subscript 0 will denote the function corresponding to the reference system. The effect of the perturbation is accurately determined if the derivatives like $g_{n}(r)$ are obtained up to sufficient orders. Traditional perturbation theory is mainly limited to the first two orders [7], however, $\mathrm{CPE}$ accounts for quite higher orders by taking recourse to the OZE, which defines the relation between the short ranged direct correlation function $c(r, \lambda)$ and the long ranged total correlation function $h(r, \lambda)$. The latter is the appropriate function to be used in the OZE as it tends to zero for large $r$, just like the pair-potential. For one-component systems with spherically symmetric potential, as in the present case, the OZE is written as:

$$
h(r, \lambda)=c(r, \lambda)+\rho \int c\left(\left|\vec{r}-\vec{r}^{\prime}\right|, \lambda\right) h\left(r^{\prime}, \lambda\right) d \vec{r}^{\prime} .
$$

where $\rho$ is the number density of atoms. This relation has to supplemented with a closure relation involving $h(r, \lambda), c(r, \lambda)$ and the pair-potential $U(r, \lambda)$. An exact closure in unfeasible, however, several approximate forms are available [3]. All the closures are generally expressed in terms of a 'bridge function' $B(r, \lambda)$ in the form:

$$
g(r, \lambda)=\operatorname{Exp}[-\beta U(r, \lambda)+y(r, \lambda)+B(r, \lambda)],
$$

where $\beta=\left(k_{B} T\right)^{-1}, k_{B}$ Boltzmann's constant and $T$ absolute temperature. Note that $k_{B} T$ is also scaled with energy unit $\mathcal{E}_{0}$. Approximate forms of $B(r, \lambda)$ generate different closures; for instance, the one called HNC mentioned earlier corresponds to the choice $B(r, \lambda)=0$.

Being an integral equation with displacement kernel, the OZE is easily solved in the Fourier space leading to the solution

$$
\bar{h}(k, \lambda)=\bar{c}(k, \lambda)[1-\rho \bar{c}(k, \lambda)]^{-1} .
$$

where the Fourier transform and its inverse, for any function $q(r)$, are defined as

$$
\bar{q}(k)=4 \pi \int_{0}^{\infty}[\sin (k r) /(k r)] q(r) r^{2} d r, \quad q(r)=\frac{1}{2 \pi^{2}} \int_{0}^{\infty}[\sin (k r) /(k r)] \bar{q}(k) k^{2} d k .
$$

A 'bar' is used throughout to denote Fourier transforms. It is useful to rewrite these equations in terms of the functions $[r q(r)]$ and $[k \bar{q}(k)]$ and the symmetric kernel $[\sin (k r)]$, as discussed below.

The two algebraic equations Eqs.(17) and (18), although defined in two different spaces, define the non-linear systems which determine the correlation functions, and entire thermodynamic properties. When the inter-particle potential contains an attractive component, solutions to the OZE with most of the closures develop singularities in the liquid-vapor transition region [14]. So determination of the critical points and phase diagrams is a difficult task, and very careful strategies need to be implemented [28]. The CPE technique completely avoids this problem of dealing with singularities, as all correlation functions are expanded in Taylor's series. The issues related to convergence of the series and 'no-solution-region' are discussed later. The Newton-Armijo non-linear solver considered next is applicable to general potentials, however, it is discussed below for the case of reference potential as required in the CPE method.

\subsection{Newton-Armijo solver for reference system}

For repulsive inter-particle potentials, as in the case of reference system, an accurate form of bridge function [27] is $B_{0}=\sqrt{1+2 y_{0}}-y 0-1$, so the closure is expressed as

$$
c_{0}(r)=\operatorname{Exp}\left[-\beta U_{R}(r)+\sqrt{1+2 y_{0}(r)}-1\right]-y_{0}(r)-1 .
$$


The solution to the OZE in terms of $\bar{y}_{0}(k)$ is given by

$$
\bar{y}_{0}(k)=\rho \bar{c}_{0}^{2}(k)\left[1-\rho \bar{c}_{0}(k)\right]^{-1} .
$$

These nonlinear equations are readily solved using Newton's method [29]. While in this reference these are treated as two equations and two unknowns, it is possible to take Eq.(20) as providing $c_{0}$ if $y_{0}$ is given. So only Eq.(21) is to be solved by Newtons' method. Starting with initial guess solution $y_{0}$ (and hence $c_{0}, \bar{c}_{0}$ and $\bar{y}_{0}$ ), an improved solution $y_{0}+\Delta y$ is obtained using the first order correction terms:

$$
\Delta c=\left[h_{0}+g_{0} B_{0}^{\prime}\right] \Delta y \quad \text { and } \quad \Delta \bar{y}=\left[\rho \frac{2 \bar{c}_{0}+\bar{y}_{0}}{1-\rho \bar{c}_{0}}\right] \Delta \bar{c}+\left[\rho \frac{\bar{c}_{0}^{2}}{1-\rho \bar{c}_{0}}-\bar{y}_{0}\right] .
$$

where $B_{0}^{\prime}$ is the derivative with respect to $y_{0}$. Taking Fourier transform of $\Delta c$ and substituting in the second equation yields

$$
\Delta \bar{y}=\rho \frac{2 \bar{c}_{0}+\bar{y}_{0}}{1-\rho \bar{c}_{0}} \overline{\left[\left(h_{0}+g_{0} B_{0}^{\prime}\right) \Delta y\right]}+\left[\rho \frac{\bar{c}_{0}^{2}}{1-\rho \bar{c}_{0}}-\bar{y}_{0}\right] .
$$

The linear operator $\mathcal{A}$ defining this equation involves the following operations: (i) Fourier inverse taking $\Delta \bar{y}$ to $\Delta y$, (ii) multiplication by $a=h_{0}+g_{0} B_{0}^{\prime}$, (iii) Fourier transform of the resulting product, and (iv) multiplication by $\bar{b}=\rho\left(2 \bar{c}_{0}+\bar{y}_{0}\right) /\left(1-\rho \bar{c}_{0}\right)$. This may be symbolically written as: $\mathcal{A} \Delta \bar{y}=$ $\Delta \bar{y}-\bar{b} \mathcal{F}\left[a \mathcal{F}^{-1}[\Delta \bar{y}]\right]$. Thus the action of $\mathcal{A}$ essentially involves two Fourier transform operations, which is re-defined with a symmetric kernel as:

$$
\left.\bar{Q}(k)=4 \pi \int_{0}^{\infty}[\sin (k r)] Q(r)\right] d r, \quad Q(r)=\frac{1}{2 \pi^{2}} \int_{0}^{\infty}[\sin (k r)] \bar{Q}(k) d k .
$$

where $Q(r)=r q(r)$ and $\bar{Q}(k)=k \bar{q}(k)$. Then the operator $\mathcal{A}$ is re-defined as $\mathcal{A}[\Delta \bar{Y}]=[\Delta \bar{Y}]-$ $\bar{b} \mathcal{S}[a \mathcal{S}[\Delta \bar{Y}]]$, where $\mathcal{S}$ represents integration with the symmetric kernel $[\sin (k r)]$. The adjoint of $\mathcal{A}$, needed in Krylov space-based linear equation solvers discussed next, is then easily computed as $\mathcal{A}^{\dagger}[\Delta \bar{Y}]=[\Delta \bar{Y}]-\mathcal{S}[a \mathcal{S}[\bar{b} \Delta \bar{Y}]]$

Numerical implementation of this algorithm uses a uniformly spaced discrete coordinates $\left\{r_{i}=i \delta r\right\}, 1 \leq i \leq N_{m}$ with $N_{m}=2^{N_{u}}$ where $N_{u}$ is, typically, 10. This choice allows the use of Fast-Fourier-transforms techniques. With a typical $\delta r \sim 0.025$, the discrete mesh extends sufficiently to account for the asymptotic variation of the correlation functions. In Fourier space, $\delta k$ is chosen such that $\delta k \delta r=\pi / N_{m}$ so that orthogonality of trigonometric functions is maintained in the discrete space. With these choices, the linear equation for $\Delta \bar{y}$ is reduced to a matrix equation of order $N_{m}$, which is most efficiently solved using Krylov space-based methods [12].

The process of improving the solution, called Newton's iterations, is continued until the Euclidean norm $\|\Delta \bar{Y}\|$ is less than a prescribed value. If the norms satisfy the condition $\|\Delta \bar{Y}\|_{1}<(1-\epsilon)\|\Delta \bar{Y}\|_{0}$ (subscripts ' 1 ' and ' 0 ' denote the current and previous iteration values, and $\epsilon \sim 10^{-4}$ is a small number), next Newton's iteration follows. Otherwise, it is likely that iteration with full step $\|\Delta \bar{Y}\|$ would diverge. Note that $\|\Delta \bar{Y}\|_{1}$ corresponds to $\mu_{1}=1$ while $\|\Delta \bar{Y}\|_{0}$ to $\mu_{0}=0$. Armijo's rule restricts the new step size to $\mu\|\Delta \bar{Y}\|$ with a value of $\mu \leq 1 / 2$. For choosing that $\mu$, a sub-iteration yielding $\|\Delta \bar{Y}\|_{2}$ with $\mu_{2}=1 / 2$ is performed. A quadratic fit of $\|\Delta \bar{Y}\|^{2}$ versus $\mu$ is then used to get $\mu_{3}$, which corresponds to the minimum of $\|\Delta \bar{Y}\|^{2}$ in the interval $\left[0.1 \mu_{2}, 0.5 \mu_{2}\right]$. Another sub-iteration providing $\|\Delta \bar{Y}\|_{3}$ is performed, and if it satisfies the norm-reduction criterion, next Newton's iteration follows. Otherwise more sub-iterations are performed, each time with the replacements $\mu_{2} \rightarrow \mu_{1}$ and $\mu_{3} \rightarrow \mu_{2}$, and similarly the respective norms. If the sub-iterations exceed a prescribed limit $(\sim 10)$, it would be necessary to restart Newton's iterations with a new guess solution.

The linear equations to be solved, at each Newton's iteration, is of the standard form $\mathcal{A} \vec{x}=\vec{b}$. The conjugate gradient (CG) method is the simplest of all iterative methods attempting a solution in 
the Krylov space, although it is applicable to symmetric matrices [30]. For non-symmetric matrices, as in the present case, the CG method is applicable to the normal equation $\mathcal{A}^{+} \mathcal{A} \vec{x}=\mathcal{A}^{\dagger} \vec{b}$ as $\mathcal{A}^{\dagger} \mathcal{A}$ is symmetric. The algorithm starts with a guess solution $\vec{x}_{0}=\vec{b}$ and its error vector $\vec{e}_{0}=\vec{b}-\mathcal{A} \vec{x}_{0}$, and an auxiliary vectors $\vec{p}_{0}=\mathcal{A}^{\dagger} \vec{e}_{0}$. Then the iteration process, called CGNR (see appendix), generates new solutions $\left\{\vec{x}_{n}\right\}$ such that the residual error norm $\left\|\vec{e}_{n}\right\|=\left\|\vec{b}-\mathcal{A} \vec{x}_{n}\right\|$ is minimized at each iteration $n$. The process converges to the exact solution in utmost $\mathcal{N}_{m}$ (dimension of the matrix) iterations if $\mathcal{A}$ is non-singular [30]. In practice, iterations are terminated when error norm $\left\|\vec{e}_{n}\right\|$ satisfies a prescribed criteria. There is little point in getting high degree of convergence in the early stages of Newton's iterations. In what are called inexact methods, the criteria used is $\left\|\vec{e}_{n}\right\| \leq \xi\|\Delta \bar{Y}\|$ with a typical value $\xi \sim 0.9$. A more common method called GMRES (generalized minimum residual), although does not need $\mathcal{A}^{\dagger}$, requires much larger storage space [30].

The correlation functions of the reference system are determined with the methods discussed (also see appendix) in the entire fluid-phase-plane except at very low temperatures, where perturbation theories are inapplicable.

\subsection{Derivatives of correlation functions}

The next step in CPE is to compute the derivatives like $g_{n}(r)$ in Eq.(15). Writing the the general closure relation in Eq.17) in the form $g=\exp [f]$, where $f=-\beta U+y+B$, repeated differentiation with respect to $\lambda$ yields

$$
g_{n}=g f_{n}+\sum_{m=1}^{n-1} C_{m}^{n-1} f_{n-m} g_{m}, \quad f_{n}=-\beta U_{A} \delta_{n 1}+y_{n}+B_{n} . \quad n \geq 1 .
$$

where $C_{m}^{n}$ denote the Binomial coefficients. This is valid for arbitrary $\lambda$, and in particular for $\lambda=0$. The arguments $r$ and $\lambda$ of all functions are omitted for simplifying the equations. Note that $U_{A}$ is the attractive component in the potential according to the WCA prescription. The summation in Eq.(25) contains only derivatives lower than $n$ as the term containing $y_{n}$ is separated out. Substituting for $f_{n}$ and using the relation $c_{n}=g_{n}-y_{n}$ for $n \geq 1$ yields the recursion relation:

$$
\begin{aligned}
c_{n} & =[(g-1)+g \Lambda] y_{n}+w_{n}, n \geq 1 \\
w_{n} & =-g \beta U_{A} \delta_{n 1}+g B_{n}^{*}+\sum_{m=1}^{n-1} C_{m}^{n-1}\left[\beta U_{A} \delta_{n-m, 1}+(1+\Lambda) y_{n-m}+B_{n-m}^{*}\right]\left[y_{m}+c_{m}\right], n \geq 1 .
\end{aligned}
$$

The derivative $B_{n}$ is written as $B_{n}=\Lambda y_{n}+B_{n}^{*}$ so that $B_{n}^{*}$ contains only derivatives of $y_{n}$ of order lower than $n$. This is needed as $y_{n}$ is yet to be determined with another equation. The definition of structure factor:

$$
\bar{s}(k, \lambda)=[1-\rho \bar{c}(k, \lambda)]^{-1}=1+\rho \bar{h}(k, \lambda),
$$

shows that $\bar{y}_{n}=\rho^{-1} \bar{s}_{n}-\bar{c}_{n}$ for $n \geq 1$. From now on the arguments $k$ and $\lambda$ of all Fourier functions are also omitted. Repeated differentiation of the relation $\bar{s}=1+\rho \bar{c} \bar{s}$ yields

$$
\bar{s}_{n}=\rho \bar{s}_{n} \bar{c}+\rho \bar{s} \bar{c}_{n}+\rho \sum_{m=1}^{n-1} C_{m}^{n} \bar{c}_{n-m} \bar{s}_{m}, n \geq 1 .
$$

where the first and last terms are separately written from the summation. Simplifying the expression and substituting in $\bar{y}_{n}$ gives the recursion

$$
\bar{y}_{n}=\left[\bar{s}^{2}-1\right] \bar{c}_{n}+\bar{q}_{n}, n \geq 1, \quad \bar{q}_{n}=\bar{s} \sum_{m=1}^{n-1} C_{m}^{n} \bar{c}_{n-m} \rho\left[\bar{y}_{m}+\bar{c}_{m}\right] n \geq 1 .
$$


Here the definition of $\bar{s}$ is used and $\bar{s}_{m}$ is substituted as $\left(\bar{y}_{m}+\bar{c}_{m}\right)$ in the summation. Note that $\bar{q}_{n}$ contains only lower order derivatives $\bar{y}_{m}$ and $\bar{c}_{m}$ for $m<n$. Taking Fourier transform of Eq.(26) and substituting in (29) yields a linear equation for $\bar{y}_{n}$ which is expressed as

$$
\bar{y}_{n}=\left[\bar{s}^{2}-1\right] \overline{\left[(h+g \Lambda) y_{n}\right]}+\left[\bar{s}^{2}-1\right] \bar{w}_{n}+\bar{q}_{n}, \quad h=g-1, \quad n \geq 1 .
$$

This expression is valid for arbitrary value of $\lambda$. The linear operator in this equation ( for all $n$ ) is identical to that in Eq.(23) if the derivatives are evaluated at $\lambda=0$, that is, for the reference system. Thus the Krylov space-based methods mentioned above are applicable here as well; the only change being the source term $\left[\bar{s}_{0}^{2}-1\right] \bar{w}_{n}+\bar{q}_{n}$.

\subsection{Derivatives of bridge function}

The density-dependent bridge function for a general potential with attractive component is expressed as [27]

$$
B=\sqrt{1+2 \zeta}-\zeta-1, \quad \zeta=y-\lambda \rho \beta U_{A}
$$

where all are functions of $r$ and $\lambda$. Defining $\mathcal{O}=B+\zeta+1=\sqrt{1+2 \zeta}$ yields $B_{n}=\mathcal{O}_{n}-\zeta_{n}$ for $n \geq 1$ where $\zeta_{n}=y_{n}-\delta_{n 1} \rho \beta U_{A}$. Now, repeated differentiation of the relation $\mathcal{O} \mathcal{O}_{1}=\zeta_{1}$ readily yields the recursion formula:

$$
\mathcal{O}_{n+1}=\mathcal{O}^{-1}\left[y_{n+1}-\sum_{m=0}^{n-1} C_{m}^{n} \mathcal{O}_{n-m} \mathcal{O}_{m+1}\right], n \geq 1
$$

which provides all derivatives $B_{n}$ in terms of $y_{n}$. The parameter $\Lambda$ defined earlier is now identified as $\Lambda=\mathcal{O}^{-1}-1$, and the summation term provides $B_{n+1}^{*}$.

\subsection{Thermodynamic functions}

After computing the Taylor's series for all functions to arbitrary high order, setting $\lambda=1$ provides correlation functions corresponding to the full potential. The results discussed below are obtained with $7^{\text {th }}$ order approximation, as further increase in order does not have any significant effect. Thermodynamic properties like pressure $(P)$ and inverse compressibility $\left(\chi^{-1}\right)$ are expressed as

$$
\begin{aligned}
\beta P / \rho & =1-(2 / 3)(\pi \beta \rho) \int_{0}^{\infty} g(r) U^{\prime}(r) r^{3} d r, \\
\chi^{-1} & =\beta(\partial p / \partial \rho)_{T}=1-4 \pi \rho \int_{0}^{\infty} c(r) r^{2} d r,
\end{aligned}
$$

Similar expressions hold for internal energy and chemical potential [28]. The argument $\lambda=1$ is omitted from these expressions. For computational purposes, Eq.(33) is re-expressed in terms of the 'cavity function' $Y=\exp (\beta U) g(r)$ as:

$$
\beta P / \rho-1=\rho B_{2 v}+(2 / 3) \pi \rho \int_{0}^{\infty}[\exp (-\beta U)]^{\prime}(Y-1) r^{3} d r .
$$

Here $B_{2 v}$ is the second virial coefficient for $U$, and is separated out explicitly. An excellent first order perturbation theory [31] for equation of state follows from this equation with the approximations: $U \approx U_{R}$ and $Y \approx Y_{0}$, thereby terminating the integral at $r_{\min }$. The second approximation is the basis of first order theory. The first approximation is motivated from the observation that derivative $[\exp (-\beta U)]^{\prime}$ is predominant only near $r_{\text {min }}$, where $Y_{0}-1$ is small.

The OZE does not provide exact thermodynamic consistency [3] in the sense that pressure computed from different routes does not agree each other. The inverse compressibility $\chi^{-1}$, being 
expressed in terms of the short ranged correlation function $c(r)$, is the more appropriate response function to compute pressure via integration with respect to $\rho$. The method followed here employs a fine mesh of $T$ and $\rho$ over the phase plane where $\chi^{-1}$ in computed. Then 2-D interpolation is used for intermediate values, and $P$ is obtained via integration over $\rho$ from zero. Solutions of the critical conditions $\partial_{v} P=0$ and $\partial_{v}^{2} P=0$ provide the critical point parameters and Maxwell's construction the phase diagram. Using thermodynamic relations to get other quantities, like energy and entropy, equation of state tables [32] can be generated in the expanded volume regions.
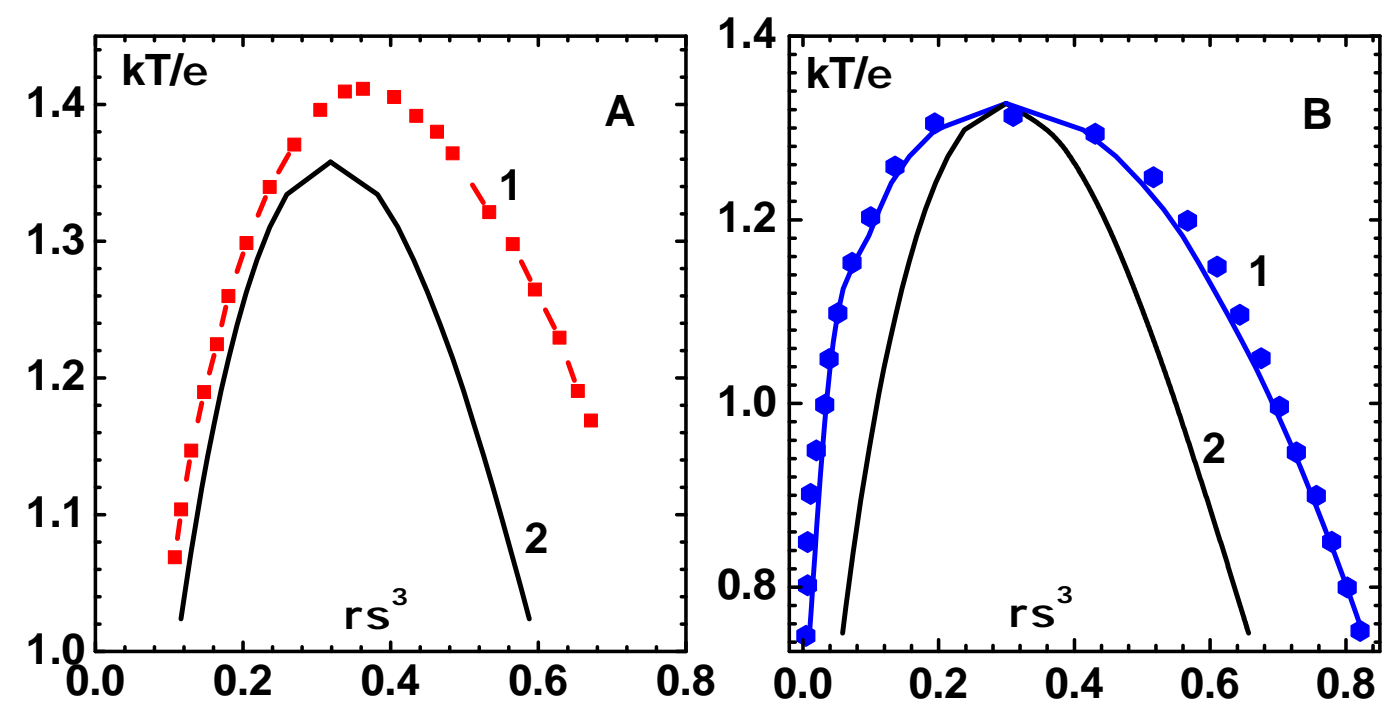

Figure 3. (A) The 'no-solution-line' (curve-1) for Lennard-Jones potential and HNC obtained with pseudo-arc-length continuation algorithm [13]. Newton's method applied to the full potential diverges on this curve. The spinodal line (curve-2) ( defined by $\chi^{-1}=0$ ) obtained using the CPE of this paper lies inside the no-solution region. (B) Comparison of co-existence line (curve-1), obtained with CPE and density-dependent bridge function [27], with simulation results (symbols) [33]. The spinodal line (curve-2), which is different from that obtained with HNC, is also shown.

\section{5. 'No-solution-region'}

As pointed out earlier, the OZE develops singularities in the liquid-vapor transition region. This problem has been investigated in detail for the Lennard-Jones (LJ) potential: $U(r)=4 \epsilon\left[(\sigma / r)^{12}-\right.$ $(\sigma / r)^{6}$, with the HNC closure [13] and other closures [14]. The pseudo-arc-length continuation algorithm is needed for direct application of Newton's method to the full potential for tracking these singularities [13]. The no-solution-line is defined as the curve in the phase plane along which the Jacobian is singular and so Newton's method is inapplicable. This curve for LJ-HNC model [13] is displayed (curve-1) in Fig.3A. In fact, there are more characteristic features like the 'fold-bifurcation' of solutions in the liquid-vapor region of the nonlinear OZE. However, CPE method does not pick up these singularities as it is based on series expansion about the repulsive component of the potential. The situation is similar that of van der Waals equation, although free of singularities, which predicts a region of negative compressibility. The spinodal line ( defined by $\chi^{-1}=0$ ) for LJ-HNC model, which is obtained using $7^{\text {th }}$ order CPE of this paper (curve-2), is also shown in Fig.3A.

The LJ system is also investigated using the density-dependent bridge function [27] for comparison with simulation data[33]. This is important because the bridge function is the sole ingredient that determines the predictive power of OZE. Results for phase diagram (co-existence curve) obtained with CPE shown (curve-1) in Fig.3B compare well with simulation data (symbols). There is only slight disagreement along the liquid branch near the transition point. The critical point parameters obtained: 
$\rho_{c} \sigma^{3}=0.299[0.312], k_{B} T_{c} / \epsilon=1.326$ [1.316] and $P_{c} \sigma^{3} / \epsilon=0.102[0.127]$ also compare well with simulation data given in square brackets.

\section{Liquid-vapor phase transition in metals}

The CPE method and inter-particle potentials derived via lattice inversion method for metallic fluids are evaluated next as these have softer repulsive components. Simulation results using Morse potential [34] with parameters corresponding to $\mathrm{Au}$ and $\mathrm{Cu}$ are used for comparing phase diagrams. The data given in Table 6 and the cohesive energy formula in Eq.(4) provide the parameter-free potentials for use in CPE. Results shown (curve-2) in Fig.4A and simulation data [34] (symbols) for $\mathrm{Au}$ agree quite well. Critical point parameters of CPE method, viz., $\rho_{c}=5.729[5.925] \mathrm{g} / \mathrm{cm}^{3}$, $T_{c}=7643[7566] \mathrm{K}$ and $P_{c}=0.598[0.525] \mathrm{GPa}$ and simulations (given in square brackets) also agree well. The spinodal curve (defined by $\chi^{-1}=0$ ) is also shown (curve-1). A similar comparison of phase diagram for $\mathrm{Cu}$ is shown (curve-2) in Fig.4B with simulation data [34] (symbols). Critical point parameters $\rho_{c}=2.309[2.631] \mathrm{g} / \mathrm{cm}^{3}, T_{\mathcal{c}}=8800[8650] \mathrm{K}$ and $P_{c}=0.905$ [0.954] GPa also compare reasonably with simulation results given in square brackets.
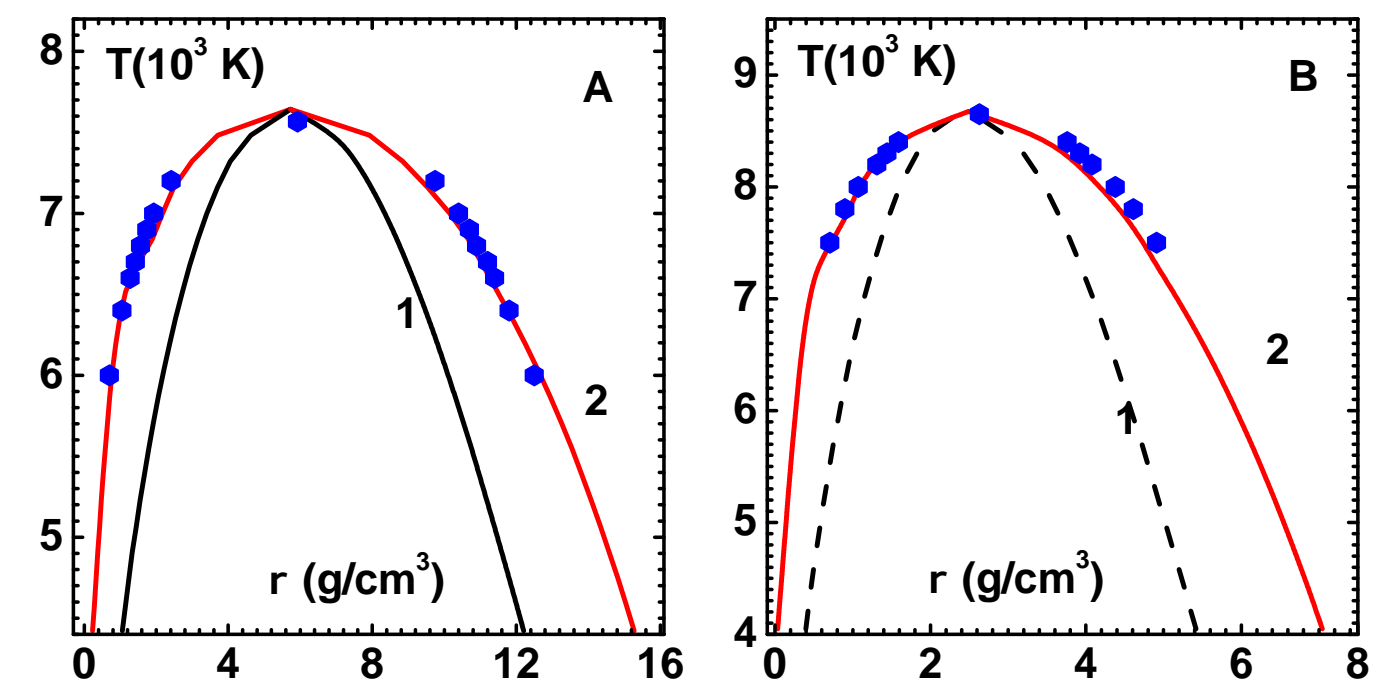

Figure 4. Comparison of phase diagram obtained with $7^{\text {th }}$ order CPE and density-dependent bridge function [27] against simulation data for soft repulsive potentials occurring in metallic fluids: (A) The $\mathrm{CPE}$ phase diagram for $\mathrm{Au}$ (curve-2) and simulation results (symbols) using Morse potential [34] (see text). The spinodal curve (defined by $\chi^{-1}=0$ ) that shows the boundary of meta-stable states (curve-1) is also shown. (B) The CPE phase diagram for $\mathrm{Cu}$ (curve-2) is compared against simulation results (symbols) using Morse potential [34] (see text). The spinodal curve (curve-1) is also shown. 

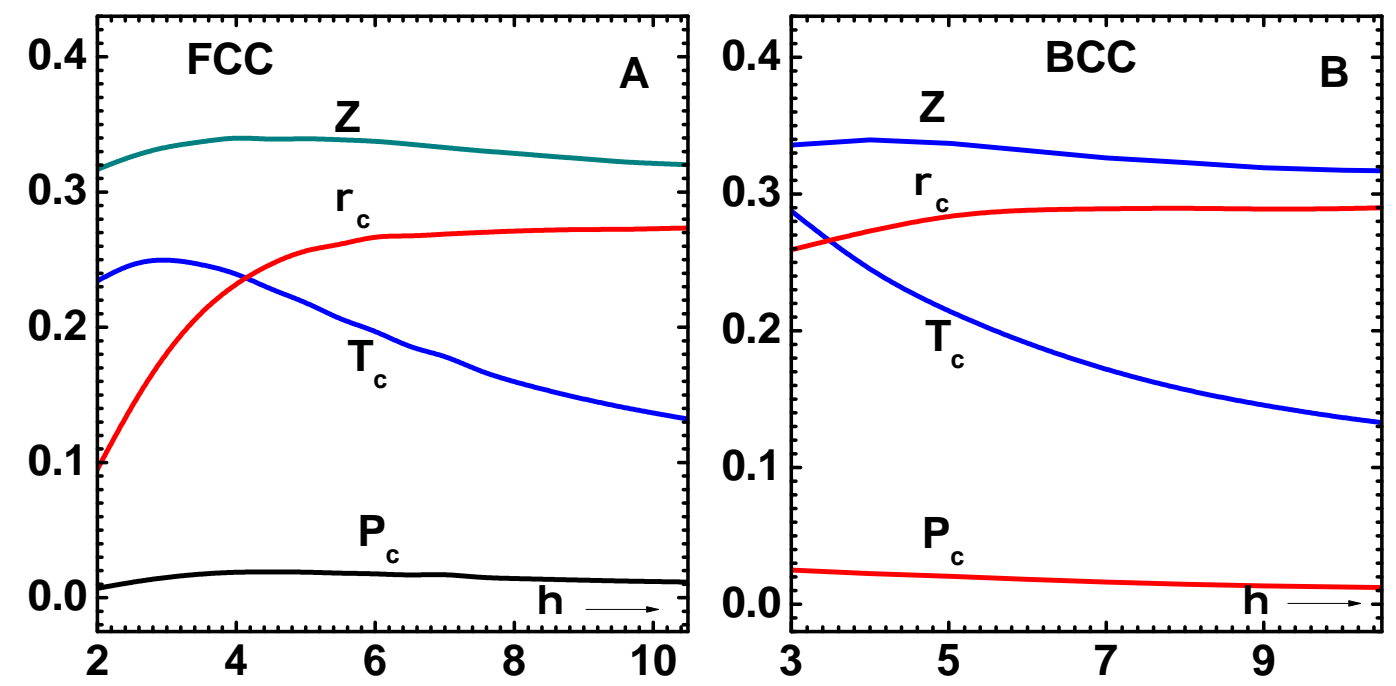

Figure 5. Variation of critical point parameters: $\rho_{\mathcal{c}}, T_{\mathcal{c}}, P_{\mathcal{c}}$ and $Z=P_{c} /\left(k_{B} T_{c} \rho_{c}\right)$, with the scaling variable $\eta$ (see text). Note that these quantities are expressed in the units: $\mathcal{V}_{0}^{-1}, \mathcal{E}_{0} / k_{B}$ and $\mathcal{E}_{0} / \mathcal{V}_{0}$, respectively. (A) Results for FCC lattices. (B) Results for BCC lattices .
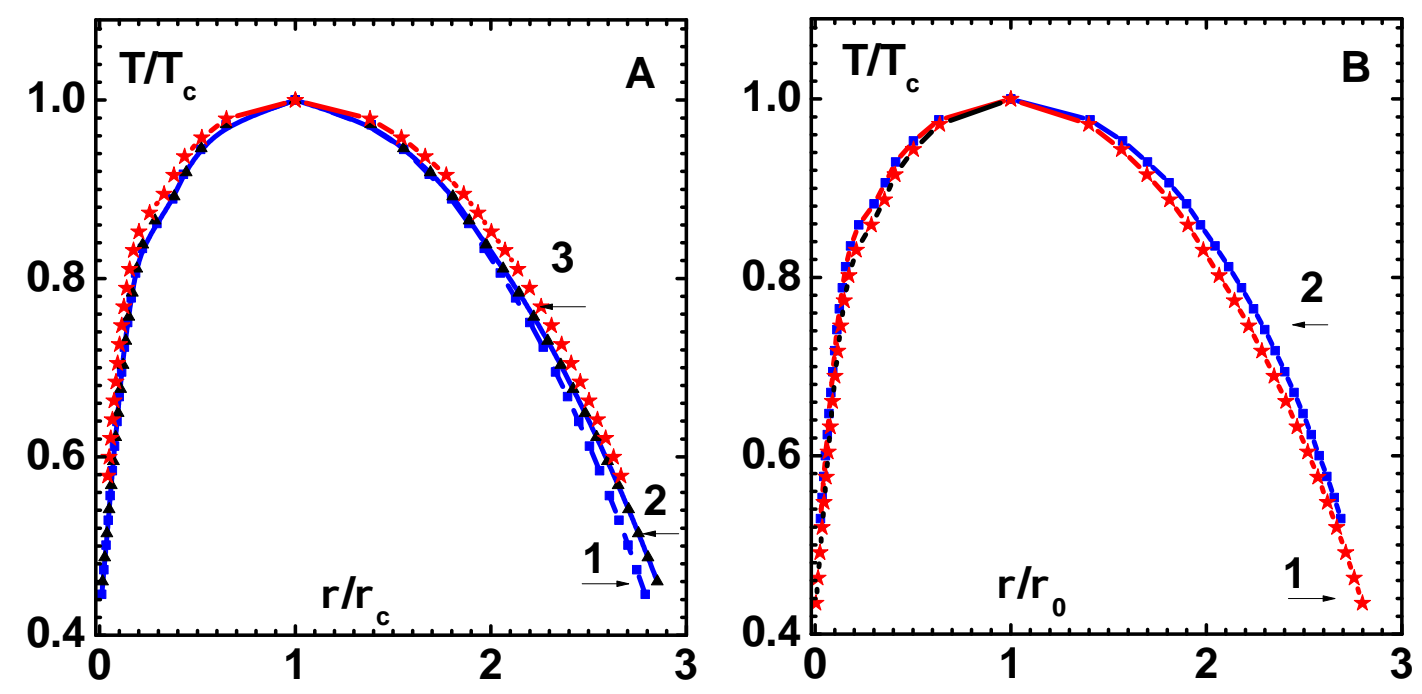

Figure 6. Phase diagram of metals in reduced variables $T / T_{\mathcal{c}}$ and $\rho / \rho_{c}$. (A) FCC metals Al (curve-1), $\mathrm{Cu}$ (curve-2) and Au (curve-3). (B) Similar results for BCC metals Fe (curve-1) and W (curve-2).

\subsection{Scaling of critical parameters and phase diagram}

Having established the accuracy of methods, scaling behavior of critical point parameters and phase diagram is now investigated. As the cohesive energy formula in Eq.(4) contains only two parameters, $(\eta$ and $\delta)$, it is obvious that the effective potentials and, hence, all the features related to co-existence also depend only on these variables. Of these two, $\delta$ is generally a small number (see Table 6). Computations using CPE is performed to determine the variation of critical point parameters: $\rho_{\mathcal{c}}, T_{\mathcal{c}}, P_{\mathcal{c}}$ and $Z=P_{\mathcal{c}} /\left(k_{B} T_{\mathcal{c}} \rho_{\mathcal{c}}\right)$, with the scaling variable $\eta$, and results for FCC lattices are shown in 
Fig.5A. Note that the graph displays dimensionless quantities expressed in the units: $\mathcal{V}_{0}^{-1}, \mathcal{E}_{0} / k_{B}$ and $\mathcal{E}_{0} / \mathcal{V}_{0}$, respectively. Similar results for BCC lattices are provided in Fig.5B. The parameter $\delta$ is kept constant at 0.0808 for FCC and 0.0148 for BCC lattices. Varying this parameter within its range does not produce significant changes in these curves. For FCC lattices the variation in $\rho_{c}$ and $T_{c}$ are more pronounced for smaller values of $\eta$. These results are somewhat similar to those obtained earlier [5], however, their variations and dependence on the lattice type are new.

Phase diagrams for three FCC metals, $\mathrm{Al}$ (curve-1), $\mathrm{Cu}$ (curve-2) and Au (curve-3) are displayed in reduced variables $T / T_{c}$ and $\rho / \rho_{c}$ in Fig.6A. Similar results are given in Fig.6B for two BCC metals $\mathrm{Fe}$ (curve-1) and $\mathrm{W}$ (curve-2). These results indicate that there are similarities in the co-existence region for these metallic fluids, although there is no perfect universality, as in the case of van der Waals model. Similar observations are also found in simulation data of phase diagrams using Morse potential [34].

\section{Summary}

The main aim in this paper is to discuss the scaling aspects of liquid-vapor phase transition in metallic fluids. There are two requirements to carry out this investigation: (i) realistic (effective) inter-particle potentials and (ii) an accurate statistical mechanical model. The four-parameter formula

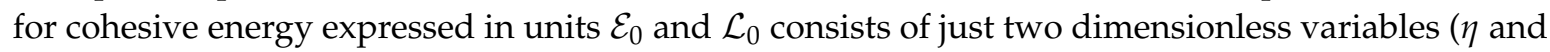
$\delta$ ). This is quite accurate in the compressed as well as expanded volume states, although needs to be corrected for extreme compression using the QSM for electrons. So effective potentials derived from the cohesive energy formula will mainly depend on these variables $\eta$ and $\delta$. Two complementary ways to effect lattice inversion are considered: (i) direct iterative method and (ii) Chen-Möbius inversion formula. New tables for inversion parameters covering up to 20 atomic shells are generated for FCC and BCC lattices, which are easily extended to other lattice types. Variations of the potential depth and first neighbor distance versus $\eta$ are then evaluated for the two lattices.

The statistical mechanical model using CPE is revisited and explicit recursion formulas for the derivatives of correlations and bridge function are derived. In fact very high order derivatives are easily computed with the present formulation. The Newton-Armijo nonlinear solver together with Krylov space-based linear solvers are implemented within the CPE. The same linear solvers are applicable to solve the linear equations for the derivatives of correlation functions, as the matrices involved are the same. With these techniques it is possible to cover the entire fluid region, except in the low temperature limit where perturbation theory is inapplicable. The entire procedure is evaluated with simulation data for LJ system as well as metallic fluids. The 'no-solution-region' concurring when OZE is applied to the full potential is also checked with CPE results for LJ system. The variations of critical ;point parameters versus $\eta$ are then evaluated for FCC and BCC lattices. Further, it is also shown that the phase diagrams of these systems show (approximate) universal behavior when expressed in terms of reduced variables. It is hoped that CPE elaborated in this paper would be increasingly used to derive thermodynamic properties of one component fluids. To that end, short outlines of the main algorithmic components are provided in the appendix. 


\section{Appendix}

\section{Algorithm for CPE}

The main components of the algorithm to implement CPE is provided below. Newton is the non-liner solver for the reference potential and CGNR is the linear solver. Other linear solvers like GMRES can also be used. Matrix computes matrix-vector products in the linear solver. Recursive equations for derivatives of correlation functions are in Derivative. The heart of the algorithm is fast Fourier transforms provided in Fourier. Note that $h_{0}^{*}$ and $\Psi$ are global variables used in Matrix.

Typical parameters

$\begin{array}{lllll}N_{u}=9 & N_{m}=2^{N_{u}} & \tau_{n w}=.0001 & \tau_{\text {in }}=.0001 & d x=.025 \\ \max N=15 & \max I=15 & \max D=7 & d k=\pi /\left(N_{m} d x\right) & \\ C_{i}=\cos \left[\pi(i-1) / N_{m}\right] & i: 1, N_{m}+1 & & & \end{array}$

\begin{tabular}{lll} 
Algorithm : & Newton $\left[y_{0}, c_{0}\right]$ & (in : $y_{0}$ out : $\left.c_{0}, y_{0}\right)$ \\
\hline$\overline{i=1, y_{0}}=0$ & While $[i \leq \max ]$ Do \{ & $g_{0}=\exp \left[-\beta U_{R}+\sqrt{\left(1+2 y_{0}\right)}-1\right]$ \\
$\Lambda=1 / \sqrt{1+2 y_{0}}-1$ & $h_{0}^{*}=g_{0}-1+g_{0} \Lambda$ & $c_{0}=g_{0}-1-y_{0}$ \\
$\bar{y}_{0}=$ Fourier $\left[1, y_{0}\right]$ & $\bar{c}_{0}=$ Fourier $\left[1, c_{0}\right]$ & $\Psi=\rho\left(\bar{y}_{0}+2 \bar{c}_{0}\right)\left[1-\rho \bar{c}_{0}\right]^{-1}$ \\
$\bar{b}=\rho \bar{c}_{0}^{2}\left[1-\rho \bar{c}_{0}\right]^{-1}-\bar{y}_{0}$ & Call CGNR $[\bar{b}, Y]$ & $\bar{y}_{0} \leftarrow \bar{y}_{0}+Y$ \\
$y_{0} \leftarrow y_{0}+$ Fourier $[2, Y]$ & if $\| k \times Y \mid<\tau_{n w}$ Exit & $i \leftarrow i+1\}$
\end{tabular}

\begin{tabular}{|c|c|c|c|}
\hline Algorithm : & CGNR $[\vec{b}, \vec{x}]$ & (in : $\vec{b}$ out : $\vec{y}$ ) & \\
\hline$\vec{x}_{0}=\vec{b}$ & $\vec{e}_{0}=\vec{b}-\mathcal{A} \vec{x}_{0}$ & $\vec{p}_{0}=\mathcal{A}^{\dagger} \vec{e}_{0}$ & $\vec{w}_{0}=\vec{p}_{0}$ \\
\hline$n=1$ & While $[n \leq \max I]$ Do \{ & $\vec{q}_{n}=\mathcal{A} \vec{p}_{n-1}$ & \\
\hline$a=\left(\vec{q}_{n} \cdot \vec{e}_{n-1}\right) /\left(\vec{q}_{n} \cdot \vec{q}_{n}\right)$ & $\vec{x}_{n}=\vec{x}_{n-1}+a \vec{p}_{n-1}$ & $\vec{e}_{n}=\vec{e}_{n-1}-a \vec{q}_{n}$ & $\vec{w}_{n}=\mathcal{A}^{\dagger} \vec{e}_{n}$ \\
\hline$\alpha=\left(\vec{w}_{n} \cdot \vec{w}_{n}\right) /\left(\vec{w}_{n-1} \cdot \vec{w}_{n-1}\right)$ & $\vec{p}_{n}=\vec{w}_{n}+\alpha \vec{p}_{n-1}$ & if $\left\|\overrightarrow{e_{n}}\right\|<\tau_{\text {in }}$ Exit & $n \leftarrow n+1\}$ \\
\hline
\end{tabular}

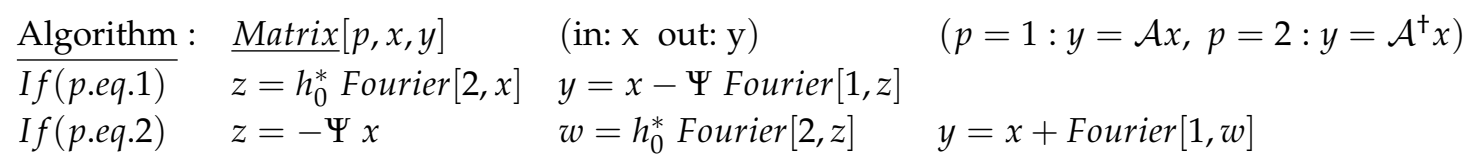

Algorithm : $\quad$ Derivative $[\max D] \quad\left(\right.$ in : $\max D$ out : $\left.y_{n}, c_{n}\right)$

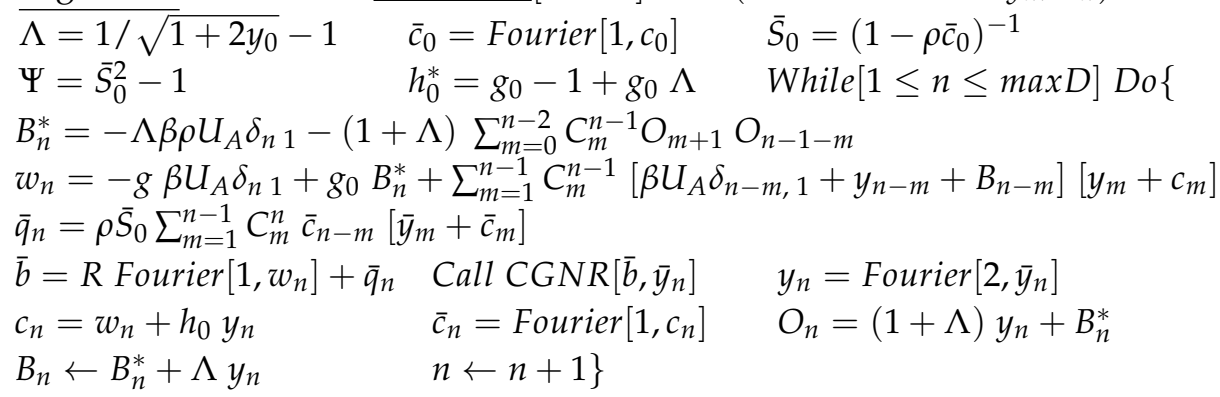




\begin{tabular}{|c|c|c|c|c|}
\hline Algorithm & $\underline{\text { Fourier }}[p, x, y]$ & (in: $\mathrm{p}, \mathrm{x}$ out: $\mathrm{y}$ ) & $c_{1}=4 \pi d x$ & $c_{2}=d k /\left(2 \pi^{2}\right)$ \\
\hline $\begin{array}{l}D o\{ \\
l=1\end{array}$ & $p=1: x_{i} \leftarrow c_{1} r_{i} x_{i}$ & $p=2: x_{i} \leftarrow c_{2} k_{i} x_{i}$ & $\left.\left[i: 1, N_{m}-1\right]\right\}$ & \\
\hline Do \{ & $m_{1}=N_{m} / l$ & $m_{2}=m_{1} / 2$ & $m_{5}=0$ & $m_{6}=m_{2}$ \\
\hline Do \{ & $i i=1$ & $s u m=0$ & & \\
\hline Do \{ & $s u m=s u m+i i \times x_{m_{5}+i}$ & $i i=-i i$ & $\left.\left[i: 1, m_{1}-1,2\right]\right\}$ & $y_{m_{6}}=s u m$ \\
\hline$D o\{$ & $i_{2}=m_{5}+2 i$ & $y_{m_{5}+i}=x_{i_{2}-1}+x_{i_{2}+1}$ & $y_{m_{6}+i}=x_{i_{2}}$ & $\left.\left[i: 1, m_{2}-1\right]\right\}$ \\
\hline$D o\{$ & $x_{m_{5}+i}=y_{m_{5}+i}$ & $x_{m_{6}+i}=y_{m_{6}+i}$ & $\left.\left[i: 1, m_{2}-1\right]\right\}$ & \\
\hline $\begin{array}{l}x_{m_{6}}=y_{m_{6}} \\
l=l\end{array}$ & $\begin{array}{l}m_{5}=m_{5}+m_{1} \\
\left.\left[m: 1, N_{u}-1\right]\right\}\end{array}$ & $m_{6}=m_{6}+m_{1}$ & $\left.\left[l_{1}: 1, l\right]\right\}$ & \\
\hline \multicolumn{5}{|l|}{$l=2^{N_{u}-2}$} \\
\hline Do \{ & $m_{1}=N_{m} / l$ & $m_{2}=m_{1} / 2$ & $m_{5}=0$ & $m_{6}=m_{2}$ \\
\hline Do \{ & $m_{7}=m_{6}+m_{2}$ & $i c=l$ & & \\
\hline $\begin{array}{l}D o\{ \\
i c=i c+l\end{array}$ & $\begin{array}{l}{\left[t_{1}=x_{m_{5}+i} /\left(2 C_{i c+1}\right)\right.} \\
\left.\left[i: 1, m_{2}-1\right]\right\}\end{array}$ & $t_{2}=x_{m_{6}+i}$ & $y_{m_{5}+i}=t_{1}+t_{2}$ & $y_{m_{7}-i}=t_{1}-t_{2}$ \\
\hline Do \{ & $x_{m_{5}+i}=y_{m_{5}+i}$ & $x_{m_{7}-i}=y_{m_{7}-i}$ & $\left.\left[i: 1, m_{2}-1\right]\right\}$ & \\
\hline $\begin{array}{l}m_{5}=m_{5}+m_{1} \\
l=l / 2\end{array}$ & $\begin{array}{l}m_{6}=m_{6}+m_{1} \\
\left.\left[m: 1, N_{u}-1\right]\right\}\end{array}$ & $\left.\left[l_{1}: 1, l\right]\right\}$ & & \\
\hline$D o\{$ & $\begin{array}{l}p=1: y_{i} \leftarrow y_{i} / k_{i} \\
* * * * *\end{array}$ & $\begin{array}{l}p=2: y_{i} \leftarrow y_{i} / r_{i} \\
* * * * *\end{array}$ & $\begin{array}{l}\left.\left[i: 1, N_{m}-1\right]\right\} \\
* * * * *\end{array}$ & \\
\hline
\end{tabular}

\section{References}

1. $\quad$ L. D. Landau and E. M. Lifshitz, Statistical Physics Pergamon Press, London. 1959.

2. S. V. G. Menon, Renormalization Group Theory of Critical Phenomena Wiley Eastern Ltd, India. 1995. (Available at: https:/ /www.researchgate.net/publication/260165199)

3. J.P. Hansen and I.R. McDonald, Theory of Simple Liquids (Forth Edition) Academic Press, Cambridge. 2013.

4. J.H. Rose, J.R. Smith, F. Guinea and F. Ferrante, Universal features of the equation of state of metals Phys. Rev. 1984, B 29, 2963.

5. Y. Rosenfeld, Predicting the liquid-vapor critical point from the crystal anharmonicity Contrib. Plasma Phys. 2001, 41, 183.

6. G. I. Kerley, Perturbation theory and the thermodynamic properties of fluids. II J. Chem. Phys. 1980, $73,478$.

7. J. R. Solana, Perturbation theories for thermodynamic properties of fluids and solids CRC Press, New York. 2013

8. B. J. Cowen and J. H. Carpenter, Improved reference system for corrected rigid spheres equation of state model J. Appl. Phys. 2020, 128, 055901.

9. J.H. Li, S.H. Liang, H.B. Guo, B.X. Liu, Four-parameter equation of state and determination of the thermal and mechanical properties of metals J. Alloys and Compounds $2007,431,23$.

10. A. Sai Venkata Ramana and S. V. G. Menon, Coupling-parameter expansion in thermodynamic perturbation theory Phys. Rev. 2013, E 87, 022101.

11. J.D. Weeks, D. Chandler and H.C. Andersen, Role of Repulsive Forces in Determining the Equilibrium Structure of Simple Liquids J. Chem. Phys. 1971, 54, 5237.

12. C. T. Kelley, Solving nonlinear equations with Newton's method, Fundamentals of Algorithms SIAM, Philadelphia 2003.

13. A. T. Peplow, R. E. Beardmore and F. Bresme, Algorithms for the computation of solutions of Ornstein-Zernike Equation Phys. Rev. 2006, E 74, 046705.

14. D. A. Tikhonov and G. N. Sarkisov, Singularities of solution of the Ornstein-Zernike Equation within the gas-liquid transition region Russian J. Phys. Chem. 2000, 74, 470.

15. M. J. Gillan, D. Alfe, J. Brodholt, L. Vocadlo and G. D. Price, First-principles modeling of Earth and planetary materials at high pressure and temperatures Rep. Prog. Phys. 2006, 69, 2365. 
16. N.N. Kalitkin and L.V. Kuz'mina, Curves of cold compression at high pressures Sov. Phys.-Solid state 1972, 13, 1938 .

17. R.M. More, Quantum-statistical model for high-density matter Phys. Rev. 1979, A 19, 1234.

18. G.I. Kerley, User's Manual for PANDA II- A computer code for calculating equation of state. Sandia Report, SAND88-229.UC-405 1991

19. T. A. Heltemes and G. A. Moses, BADGER v 1.0: A Fortran equation of state library Comp. Phys. Commun. 2012, 183, 2629.

20. A. E. Carlsson, C. D. Gelatt Jr and H. Ehrenreich, An ab initio pair potential applied to metals Phil. Mag. 1980, A $41,241$.

21. M. Z. Bazant and E. Kaxiras, Modeling of covalent bonding in solids by inversion of cohesive energy curves Phys. Rev. Lett. 1996, 77, 4370.

22. J. D. Wiley and J. A. Seman, The enumeration of neighbors on cubic and hexagonal-based lattices The Bell System Technical Journal 1970, March, 355.

23. Chen Nan-xian, Chen Zhao-dou and Wei Yu-chuan, Multidimensional inverse lattice problem and a uniformly sampled arithmetic Fourier transform Phy. Rev. 1997, E 55, R5.

24. Qian Xie, Wen-qing Zhang and Nan-xian Chen, Analytical long-range embedded atom potentials Phy. Rev. 1984, B $29,6443$.

25. S. M. Foiles, Application of the embedded -atom method to liquid transition metals arXiv:cond-mat 1997, [cond-mat.mtrl-sci], $9610183 v 2$.

26. S. M. Foiles, Application of the embedded-atom method to liquid transition metals Phy. Rev. 1985, B 32, 3409.

27. G. N. Sarkisov, Structure of simple fluid in the vicinity of the critical point: approximate integral equation theory of liquids J. Chem. Phys. 2003, 119, 373.

28. I. Charpentier and N. Jakse, Phase diagram of complex fluids using an efficient integral equation method J. Chem. Phys. 2005, 123, 204910.

29. G. Zerah, An efficient Newton's method for numerical solution of fluid integral equations J. Comp. Phys. 1985, 61, 280.

30. C. T. Kelley, Iterative methods for solving linear and nonlinear equations SIAM, Philadelphia 1995.

31. Y. Song and E. A. Mason, Statistical-mechanical theory of a new analytical equation of state J. Chem. Phys. 1989, 91, 7840.

32. S. V. G. Menon and Bishnupriya Nayak, An equation of state for metals at high temperature and pressure in compressed and expanded volume regions Condensed Matter $2019,4,71$.

33. J. K. Johnson, J. A. Zollweg and K. E. Gubbins, The Lennard-Jones equation of state revisited Mol. Phys. 1993, 78, 591.

34. J. K. Singh, J. Adhikari and S. K. Kwak, Vapor-liquid phase co-existence curves for Morse fluids Fluid Phase Equilibria 2006, 248, 1 . 